\title{
Over 19 W Single-Mode 1545 nm Er,Yb Codoped All-Fiber Laser
}

\author{
Jiadong Wu, ${ }^{1,2}$ Chunxiang Zhang, ${ }^{3}$ Jun Liu, ${ }^{1,2}$ Ting Zhao, ${ }^{4}$ Weichao Yao, ${ }^{1}$ Pinghua Tang, ${ }^{3}$ \\ Le Zhang, ${ }^{1,5}$ and Hao Chen ${ }^{1,5}$ \\ ${ }^{1}$ Jiangsu Key Laboratory of Advanced Laser Materials and Devices, School of Physics and Electronic Engineering, \\ Jiangsu Normal University, Xuzhou, Jiangsu 221116, China \\ ${ }^{2}$ International Collaborative Laboratory of 2D Materials for Optoelectronics Science and Technology, Key Laboratory of Optoelectronic \\ Devices and Systems of Ministry of Education and Guangdong Province, College of Optoelectronic Engineering, Shenzhen University, \\ Shenzhen 518060, China \\ ${ }^{3}$ Laboratory for Micro-/Nano-Optoelectronic Devices of Ministry of Education, IFSA Collaborative Innovation Center, \\ School of Physics and Electronics, Hunan University, Changsha 410082, China \\ ${ }^{4}$ School of Electronic Engineering, Nanjing Xiaozhuang University, Nanjing, Jiangsu 211171, China \\ ${ }^{5}$ Jiangsu Collaborative Innovation Center of Advanced Laser Technology and Emerging Industry, Jiangsu Normal University, \\ Xuzhou 221116, China
}

Correspondence should be addressed to Jun Liu; liuj1987@szu.edu.cn and Hao Chen; chenhao@jsnu.edu.cn

Received 27 June 2017; Revised 15 August 2017; Accepted 24 August 2017; Published 4 October 2017

Academic Editor: Jörg Fink

Copyright (C) 2017 Jiadong Wu et al. This is an open access article distributed under the Creative Commons Attribution License, which permits unrestricted use, distribution, and reproduction in any medium, provided the original work is properly cited.

\begin{abstract}
We report a high-power cladding-pumped Er,Yb codoped all-fiber laser with truly single transverse mode output. The fiber laser is designed to operate at $1545 \mathrm{~nm}$ by the use of a pair of fiber Bragg gratings (FBGs) to lock and narrow the output spectrum, which can be very useful in generating the eye-safe $\sim 1650 \mathrm{~nm}$ laser emission through the Stimulated Raman Scattering (SRS) in silica fibers that is of interest in many applications. Two pieces of standard single-mode fibers are inserted into the laser cavity and output port to guarantee the truly single-mode output as well as good compatibility with other standard fiber components. We have obtained a maximum output power of $19.2 \mathrm{~W}$ at $1544.68 \mathrm{~nm}$ with a FWHM spectral width of $0.08 \mathrm{~nm}$, corresponding to an average overall slope efficiency of $31.9 \%$ with respect to the launched pump power. This is, to the best of our knowledge, the highest output power reported from simple all-fiber single-mode Er,Yb codoped laser oscillator architecture.
\end{abstract}

\section{Introduction}

High-power fiber lasers in the eye-safe wavelength regime around $1.5-1.6 \mu \mathrm{m}$ have attracted considerable attention in various applications such as spectroscopy, remote sensing, range finding, and free-space and satellite communications. Cladding-pumped Er, $\mathrm{Yb}$ codoped fiber laser systems provide a common approach to produce high-power laser emissions in this wavelength region owing to the commercially available efficient double clad gain fiber and high-power InGaAs laser diodes at $\sim 980 \mathrm{~nm}$ as a pump source [1-6]. In 2007, Jeong et al. achieved $297 \mathrm{~W}$ of output power at $1.567 \mu \mathrm{m}$ in an $\mathrm{Er}, \mathrm{Yb}$ codoped fiber laser, which is the highest output power achieved with the Er,Yb codoped fibers until now [1]. However, the vast majority of high-power Er, $\mathrm{Yb}$ codoped laser systems reported so far were implemented with large mode area (LMA) gain fibers supporting tens or even hundreds of modes to mitigate the harmful nonlinear effects, which, unfortunately, degrade the output laser beam quality [2-4]. In addition, many high-power laser configurations incorporate several free-space optical components, increasing the complexity of the system and thus cost of maintenance. Nevertheless, for many applications in the eye-safe wavelength range, the requirement for high output power is also accompanied by the need of good beam quality and high efficiency resulting from simple laser structures. All-fiber geometries without free-space optics can offer alignment-free, efficient, compact, and reliable laser architecture. Single-mode all-fiber Er, $\mathrm{Yb}$ codoped laser systems have naturally become an attractive candidate for achieving high output power level while maintaining good laser beam quality to meet the requirement for particular applications. Nowadays, more than $10 \mathrm{~W}$ output 


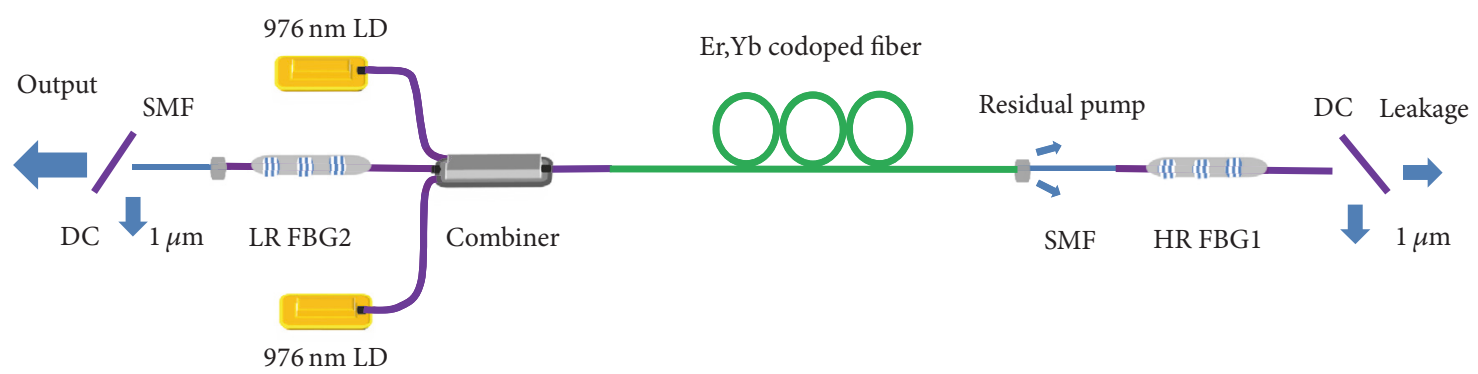

FIGURE 1: Experimental schematic of the single-mode all-fiber Er,Yb codoped laser. DC: dichroic mirror; SMF: standard single-mode fiber; FBG: fiber Bragg grating.

powers of single-mode laser emissions at $\sim 1.5 \mu \mathrm{m}$ in the $\mathrm{Er}, \mathrm{Yb}$ codoped laser systems have been achieved based on the more complex master oscillator power amplifier (MOPA) configurations $[7,8]$, while the extremely simple all-fiber $\mathrm{Er}, \mathrm{Yb}$ codoped fiber laser oscillators still remain of a limited output power level of several watts [9-11].

In this paper, we use a simple and compact cavity design with two pieces of standard single-mode fiber incorporated into the laser resonator and the output port, respectively, and demonstrate a truly single-mode high-power Er,Yb codoped all-fiber laser based on a slightly multimode gain fiber. The operating wavelength of the fiber laser is locked at $1545 \mathrm{~nm}$, which corresponds to the first-order Stokes wavelength of $1658 \mathrm{~nm}$ that can be used to precisely generate the dual mid-IR laser emissions of $2.7 \mu \mathrm{m}$ and $4.3 \mu \mathrm{m}$ through the nonlinear optical parametric frequency conversion [12-14]. A maximum overall output power of $19.2 \mathrm{~W}$ is obtained at $1544.68 \mathrm{~nm}$ with a FWHM spectral width of $0.08 \mathrm{~nm}$ and an overall slope efficiency of $31.9 \%$, limited only by the available pump power. To the best of our knowledge, this is so far the highest output power reported from all-fiber single-mode Er,Yb codoped laser oscillators.

\section{Experiment}

The experimental schematic of the single-mode all-fiber $\mathrm{Er}, \mathrm{Yb}$ codoped fiber laser is shown in Figure 1. The gain medium was a $3 \mathrm{~m}$ long Er,Yb codoped multimode double clad fiber (Nufern, MM-EYDF-12/130) with a nominal cladding-pump absorption of $3.10 \mathrm{~dB} / \mathrm{m}$ at $915 \mathrm{~nm}$, which had a core of $12 \mu \mathrm{m}$ diameter and $\sim 0.2 \mathrm{NA}$, and a pure silica innercladding of $130 \mu \mathrm{m}$ diameter and $\sim 0.46 \mathrm{NA}$ covered with a low refractive index Acrylate outcladding also as protective coating. The gain fiber had a $V$ number (cut-off frequency or normalized frequency) of 4.8, suggesting the number of laser modes that the gain fiber can support is around 11. Therefore, a piece of standard single-mode-fiber was inserted as a spatial filter into the new laser cavity with the output port made of another piece of standard single-mode fiber, in order to guarantee truly single-mode laser output and at the same time eliminate the residual pump in the cladding and other unwanted laser modes coupled into the cladding by using a high-index gel. The splicing between the unmatched fibers was carefully carried out by a specialty splicer (Fujikura,
FSM-100P+) with splicing loss of below $0.2 \mathrm{~dB}$. The heat load yielded from quantum defect over the gain fiber was dissipated by simple fan-cooling.

The lasing feedback was provided by a pair of FBGs written in a compatible single-mode double clad passive fiber (Nufern, GDF-1550). FBG1 is highly reflective with a peak reflectivity of $99 \%$ and spectral bandwidth of $0.5 \mathrm{~nm}$ at $1544.5 \mathrm{~nm}$. FBG2 is a 10\% low-reflection output coupler with the center wavelength of $1544.6 \mathrm{~nm}$ and bandwidth of $0.7 \mathrm{~nm}$, as shown in Figure 2. Due to limited effective reflectivity and slight shift of center wavelength and bandwidth for the two FBGs, as well as possible laser spectral broadening in the high-power operation, some power leakage may occur through the high-reflection FBG1 end. Thus, we also monitored the possible power leakage and corresponding spectrum from the free end facet of FBG1. Both output end facets of the fiber laser were angle-cleaved at $\sim 8^{\circ}$ to suppress the impact of Fresnel reflections from the uncoated fiber facets on the laser stability as well as the corresponding broadband feedback and then to reduce the $\sim 1 \mu \mathrm{m}$ parasitic lasing resulting from transitions of some $\mathrm{Yb}$ ions excluded from the energy transfer process between $\mathrm{Yb}$ and Er ions. Two $45^{\circ}$ dichroic mirrors with high-reflection at the laser wavelength and high-transmission in the $\sim 1 \mu \mathrm{m}$ wavelength band were inserted into the output path to steer the laser beam from the possible $\sim 1 \mu \mathrm{m}$ parasitic laser for measurement. Two commercial fiber-coupled $35 \mathrm{~W}$ wavelength stabilized $976 \mathrm{~nm}$ laser diodes were served as the pump source, the output ports of which were directly spliced to a $(2+1) \times$ 1 pump combiner to cladding-pump the gain fiber in the backward pumping regime. Both pump laser diodes were spectrally narrowed and locked at $976 \mathrm{~nm}$ by a volume Bragg grating with a $3 \mathrm{~dB}$ spectral width of $0.5 \mathrm{~nm}$ to match the absorption peak of the Er,Yb codoped fiber. The spectral characteristics of the laser output were recorded using an optical spectrum analyzer (AQ6370C, Yokogawa).

\section{Results and Discussion}

The lasing characteristics of the single-mode all-fiber Er, Yb codoped laser were evaluated. Figure 3 shows the output powers from the single-mode fiber output end as a function of the launched pump power. The laser reached threshold at a launched pump power of $\sim 1 \mathrm{~W}$ and generated an output 


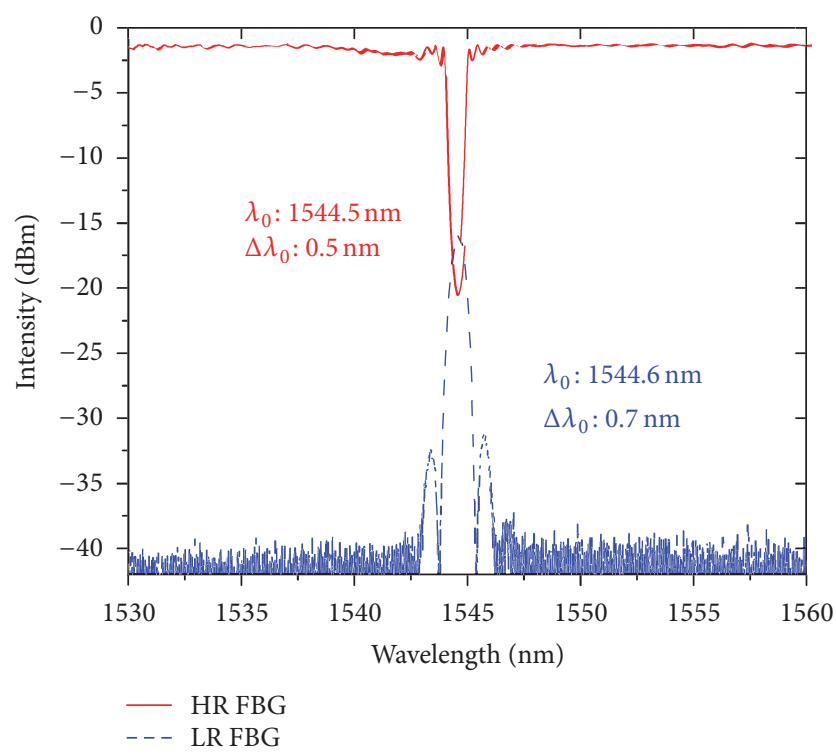

FIGURE 2: Measured transmission spectrum and reflection spectrum of the high-reflection (HR) FBG (red line) and low-reflection (LR) FBG (blue dash), respectively.

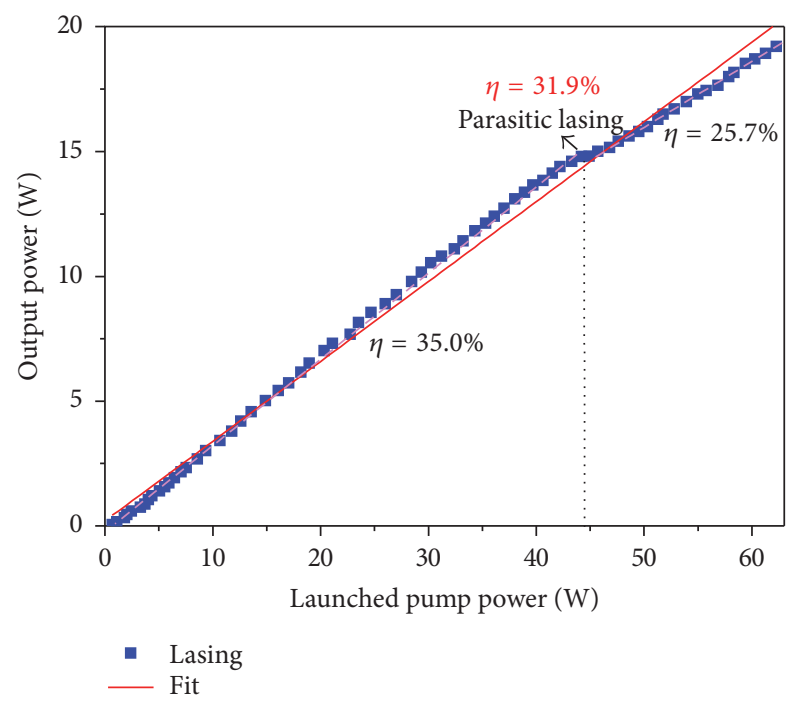

FIGURE 3: Laser output powers as a function of the launched pump power.

power of $19.2 \mathrm{~W}$ for the maximum launched pump power of $62.3 \mathrm{~W}$, corresponding to an average slope efficiency of $31.9 \%$ with respect to the launched pump power. The output laser powers showed a linear dependence with the launched pump power over the whole pump range. The output powers remained quite stable during the whole measurement. However, parasitic lasing of $\mathrm{Yb}$ at $1060.4 \mathrm{~nm}$ began to oscillate at the launched pump power of $44.2 \mathrm{~W}$, and an overall output power of $2.1 \mathrm{~W}$ was yielded at the maximum launched pump power from both fiber ends. The $\sim 1 \mu \mathrm{m}$ parasitic oscillation reduced the lasing slope efficiency from $35 \%$ to $25.7 \%$, which can be seen obviously in Figure 3. Despite this, no roll-off

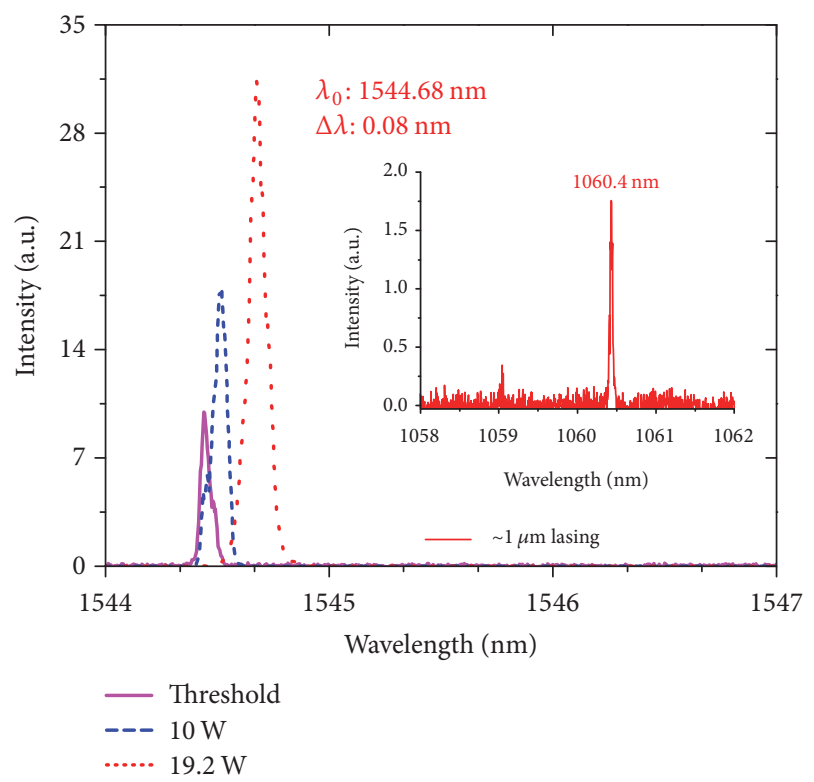

FIGURE 4: Laser output spectrum from standard single-mode fiber end and spectrum of the leaked laser from the high-reflection FBG1 end in comparison. Inset: measured spectrum of $\sim 1 \mu \mathrm{m}$ parasitic lasing.

resulting from the unfavorable nonlinear effects or thermal loading was observed. The leaked laser power from the FBG1 end was $0.2 \mathrm{~W}$, which is acceptable due to the limited effective reflectivity of the high-reflection FBG. In addition, it is worth noting that the fiber laser was operated in the backward pumping regime to avoid any gain fiber burning adjacent to the combiner that commonly occurred in the forward pumping regime due to the abrupt change of temperature gradient in this position [15].

Figure 4 shows the output spectral evolution at different laser power levels from the standard single-mode fiber output end. We can see that the laser center wavelength is redshifted from $1544.44 \mathrm{~nm}$ near the threshold to $1544.68 \mathrm{~nm}$ at the output power of $19.2 \mathrm{~W}$, which is primarily attributed to the temperature dependence of reflection wavelength of the FBGs. The spectral width was broadened from a FWHM linewidth of $0.04 \mathrm{~nm}$ to $0.08 \mathrm{~nm}$ correspondingly. Despite the spectral broadening, the oscillating laser modes still remained within the bandwidth of the two FBGs. In the lasing feedback of each round trip, FBG2 will sample a nominal $10 \%$ of the lasing components within its reflection band and reflect it back into the cavity. This portion of light will then be amplified in the backward direction. Upon reaching FBG1 the laser modes within the reflection band are highly reflected (99\% in our case) and then successively amplified in the forward direction towards FBG2, where $90 \%$ of the laser will be outcoupled while the small reflected portion returns to the next lasing cycle. In our experiment the laser leakage from FBG1 was primarily attributed to its limited effective reflectivity since the bandwidth of FBG2 is slightly larger than that of FBG1. Despite this, the leaked laser from FBG1 was measured to have roughly the same spectral profile without central dip or any spectral ripples as that from the output end 
in terms of center wavelength and width. Thus, both output beams can be simply combined for certain applications. Alternatively, we can also use a high-reflection FBG with much higher reflectivity and broader bandwidth instead to reduce the leakage power from this end.

In addition, it is worth noting that the truly single-mode laser output was actually achieved literally through first a piece of standard single-mode fiber in the cavity and then another piece of standard single-mode fiber on the output end without sacrificing much laser output power loss. Although the Er,Yb codoped gain fiber was capable of supporting 11 laser modes at $\sim 1.5 \mu \mathrm{m}$, the amount of output laser modes from the gain fiber was actually much less than specified, owing to the intense mode competition where only the modes with lowest loss survived. Besides, the standard single-mode fiber inserted in the cavity and single-mode double clad pig-tailed fiber of FBG2 connected to the Er,Yb codoped fiber further suppressed most of the higher order laser modes yielded in the gain fiber, which ensures a relatively low splicing loss with the standard single-mode fiber. The last piece of standard single-mode fiber in the output end guaranteed a truly single-mode laser output from the $1545 \mathrm{~nm}$ all-fiber laser.

\section{Conclusions}

We demonstrate a truly single-mode all-fiber high-power Er, Yb codoped laser at $1545 \mathrm{~nm}$ which is potentially significant for some applications. The laser output port is convinced with a piece of standard single-mode fiber to guarantee the truly single-mode output while keeping good compatibility with other standard fiber components. A maximum overall output power of $19.2 \mathrm{~W}$ at $1544.68 \mathrm{~nm}$ with a FWHM spectral width of $0.08 \mathrm{~nm}$ is obtained in the fiber laser, corresponding to an average slope efficiency of $31.9 \%$ with respect to the launched pump power. To the best of our knowledge, this is the highest single-mode output power achieved from a simple all-fiber Er,Yb codoped laser oscillator architecture.

\section{Conflicts of Interest}

The authors declare that there are no conflicts of interest regarding the publication of this paper.

\section{Authors' Contributions}

Jiadong Wu and Chunxiang Zhang contributed equally to the paper.

\section{Acknowledgments}

This work is partially supported by the National Natural Science Fund Foundation of China (Grant nos. 61505124 and 61505122 and 61605166), the Science and Technology Planning Project of Guangdong Province of China (Grant no. 2016B050501005), the Educational Commission of Guangdong Province (Grant no. 2016KCXTD006), and Natural Science Foundation of SZU (Grant no. 2017022).

\section{References}

[1] Y. Jeong, S. Yoo, C. A. Codemard et al., "Erbium:ytterbium codoped large-core fiber laser with $297-W$ continuous-wave output power," IEEE Journal on Selected Topics in Quantum Electronics, vol. 13, no. 3, pp. 573-578, 2007.

[2] D. Y. Shen, J. K. Sahu, and W. A. Clarkson, "Highly efficient Er, Yb-doped fiber laser with $188 \mathrm{~W}$ free-running and $>100 \mathrm{~W}$ tunable output power," Optics Express, vol. 13, no. 13, pp. 4916-4921, 2005.

[3] J. Liu, D. Shen, H. Huang, X. Zhang, X. Liu, and D. Fan, "Volume bragg grating-based tunable $\mathrm{Er}, \mathrm{Yb}$ fiber lasers covering the whole C-and L-band," IEEE Photonics Technology Letters, vol. 25, no. 15, pp. 1488-1491, 2013.

[4] J. W. Kim, P. Jelger, J. K. Sahu, F. Laurell, and W. A. Clarkson, "High-power and wavelength-tunable operation of an Er5Yb fiber laser using a volume Bragg grating," Optics Letters, vol. 33, no. 11, pp. 1204-1206, 2008.

[5] Y. Jeong, J. K. Sahu, D. B. S. Soh, C. A. Codemard, and J. Nilsson, "High-power tunable single-frequency single-mode erbium:ytterbium codoped large-core fiber master-oscillator power amplifier source," Optics Letters, vol. 30, no. 22, pp. 2997-2999, 2005.

[6] Y. Chen, G. Jiang, S. Chen et al., "Mechanically exfoliated black phosphorus as a new saturable absorber for both Q-switching and mode-locking laser operation," Optics Express, vol. 23, no. 10, pp. 12823-12833, 2015.

[7] G. Sobon, D. Sliwinska, K. M. Abramski, and P. Kaczmarek, "10 W single-mode Er/Yb co-doped all-fiber amplifier with suppressed Yb-ASE," Laser Physics Letters, vol. 11, no. 2, Article ID 025103, 2014.

[8] B. Morasse, S. Aggerb, S. Chatignya, É. Gagnona, J. Sandroa, and C. Poulsen, "10W ASE-free single-mode high-power doublecladding $\mathrm{Er}^{3+}-\mathrm{Yb}^{3+}$ amplifier," in Fiber Lasers IV: Technology, Systems, and Applications, vol. 6453 of Proceeding of the SPIE, 2007.

[9] S. Gurram, A. Kuruvilla, R. Singh, and K. S. Bindra, "Generation of $6.8 \mathrm{~W}$ of CW output power at $1550 \mathrm{~nm}$ using small mode field diameter Er:Yb co-doped double clad fiber in laser oscillator configuration," Laser Physics, vol. 27, no. 6, p. 065113, 2017.

[10] P. Peterka, I. Kašík, V. Kubeček et al., "Optimization of erbiumytterbium fibre laser with simple double-clad structure," in Proceedings of the Photonics, Devices and Systems III, vol. 6180 of Proceeding of the SPIE, 2006.

[11] M. A. Jebali, J.-N. Maran, S. LaRochelle, S. Chatigny, M. A. Lapointe, and E. Gagnon, "A $103 \mathrm{~W}$ high efficiency in-band cladding-pumped $1593 \mathrm{~nm}$ all-fiber erbium-doped fiber laser," in Proceedings of the CLEO: Science and Innovations, CLEO_SI '12, p. JTh1I.3, May 2012.

[12] J. Liu, D. Shen, H. Huang, C. Zhao, X. Zhang, and D. Fan, "Highpower and highly efficient operation of wavelength-tunable Raman fiber lasers based on volume Bragg gratings," Optics Express, vol. 22, no. 6, pp. 6605-6612, 2014.

[13] M. Wang, L. Zhu, W. Chen, and D. Fan, "Efficient all-solid-state mid-infrared optical parametric oscillator based on resonantly pumped $1.645 \mu \mathrm{m}$ Er:YAG laser," Optics Letters, vol. 37, no. 13, pp. 2682-2684, 2012.

[14] J. Liu, P. Tang, Y. Chen et al., "Highly efficient tunable midinfrared optical parametric oscillator pumped by a wavelength locked, Q-switched Er:YAG laser," Optics Express, vol. 23, no. 16, pp. 20812-20819, 2015. 
[15] C. Shi, R. T. Su, H. W. Zhang et al., "Experimental study of output characteristics of bi-directional pumping high power fiber amplifier in different pumping schemes," IEEE Photonics Journal, vol. 9, no. 3, pp. 1-10, 2017. 

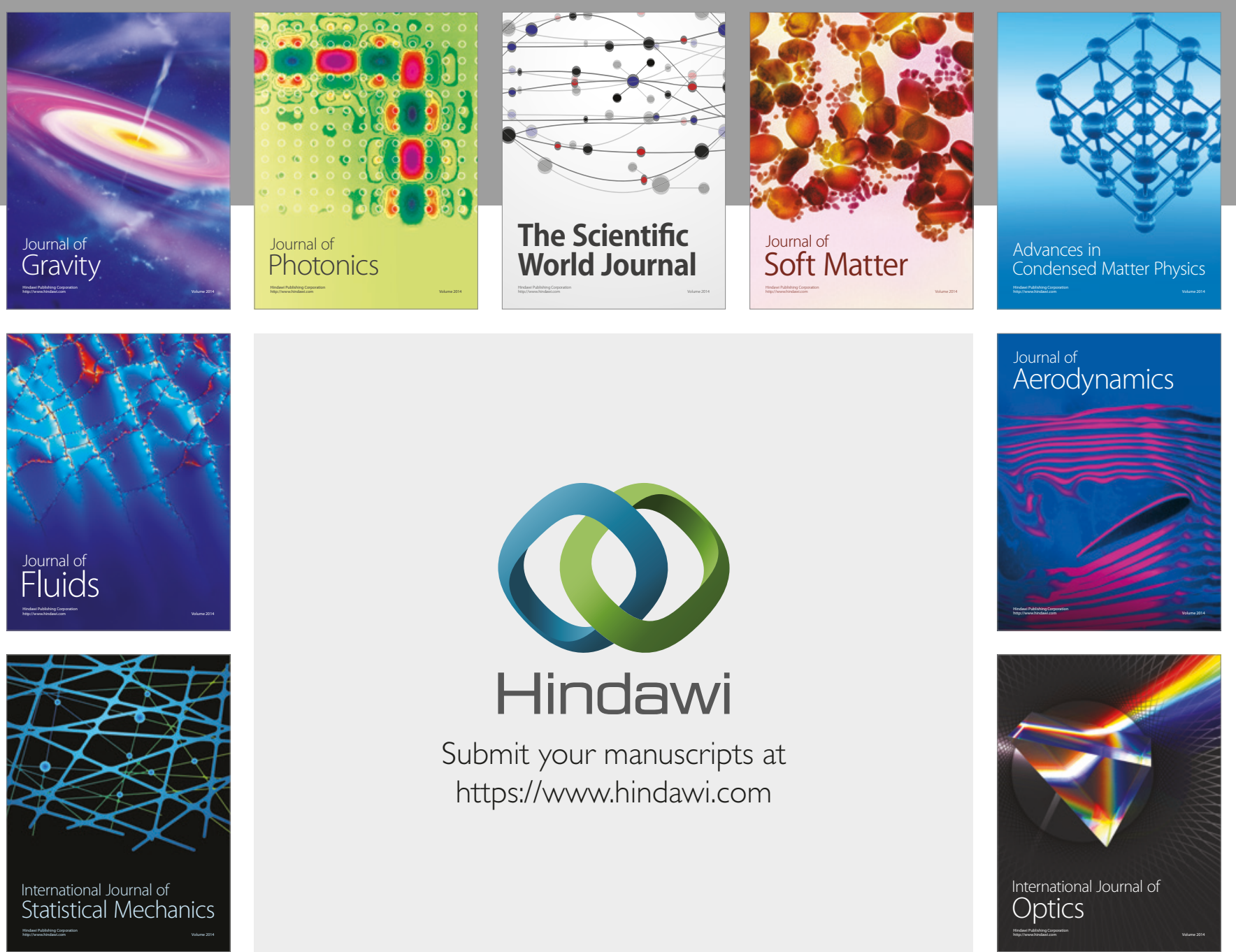

Submit your manuscripts at

https://www.hindawi.com
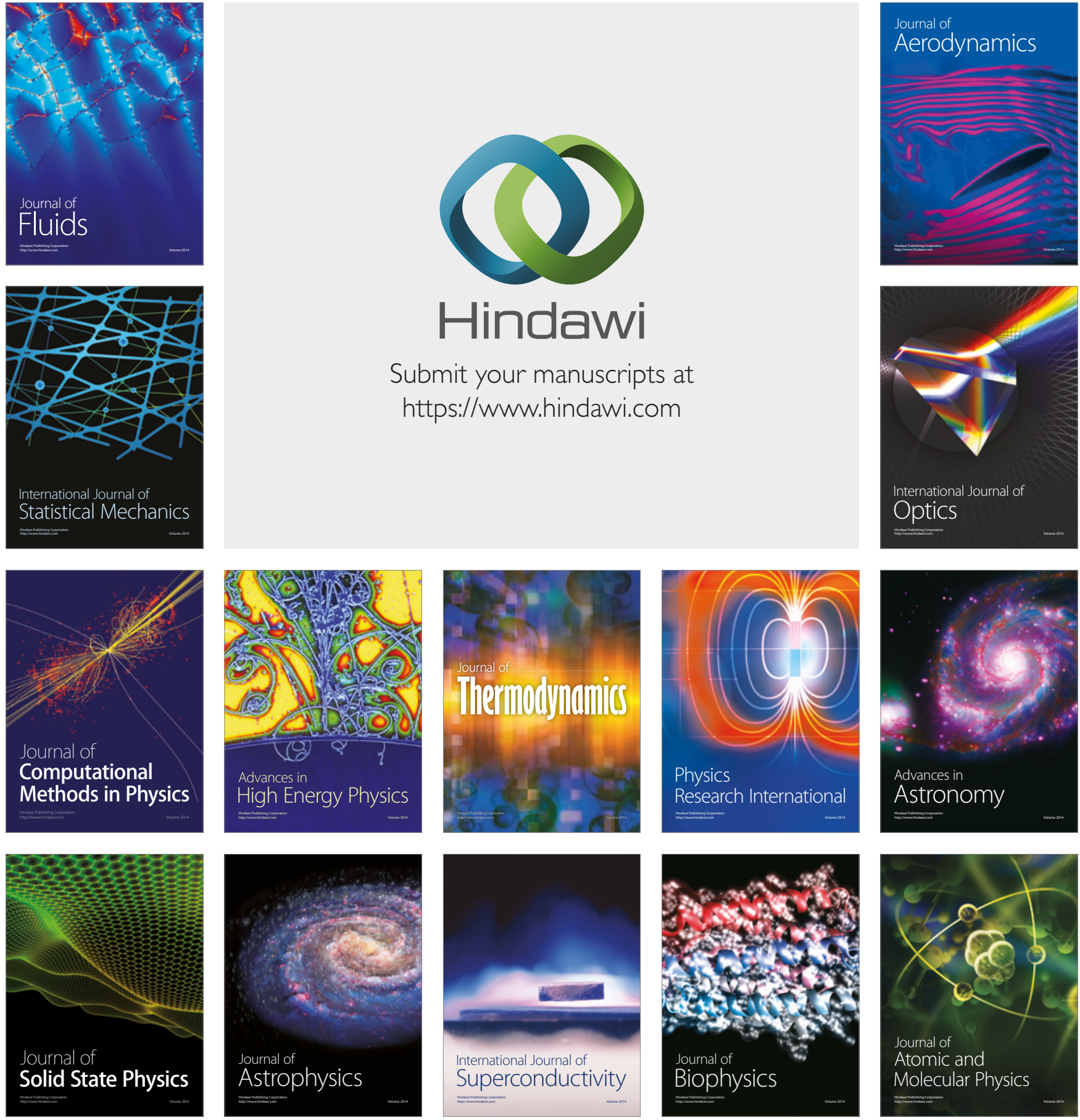